\title{
THE MODULATION OF SHORT GRAVITY WAVES BY LONG WAVES OR CURRENTS
}

\author{
R. GRIMSHAW ${ }^{1}$
}

(Received 6 May, 1987; revised 28 July 1987)

\begin{abstract}
The modulation of short gravity waves by long waves or currents is described for the situation when the flow is irrotational and when the short waves are described by linearised equations. Two cases are distinguished depending on whether the basic flow can be characterised as a deep-water current, or a shallow-water current. In both cases the basic flow has a current which has finite amplitude, while in the first case the free surface slope of the basic flow can be finite, but in the second case is small. The modulation equations are the local dispersion relation of the short waves, the kinematic equation for conservation of wave crests and the wave action equation. The results incorporate and extend the earlier work of Longuet-Higgins and Stewart $[10,11]$.
\end{abstract}

\section{Introduction}

Since the pioneering work by Longuet-Higgins and Stewart $[10,11]$ on the modulation of short gravity waves by long waves or currents, there has been a large amount of research done, much of which has been summarised in texts such as those by Whitham [18], Le Blond and Mysak [9], and Mei [14]. However, at least in the early stages of the development of this topic, the unifying nature of the general concepts of wave kinematics and wave action conservation were not fully appreciated. Hence, at a time when remote sensing techniques have given a new importance to short-wave modulation, it seems useful to re-examine and review this topic. Of course, short-wave modulation in the ocean is likely to be significantly affected by wave breaking and by the presence of wind-induced shear in the surface layer of the ocean. Although these aspects will not be addressed here,

${ }^{1}$ School of Mathematics, University of New South Wales, P.O. Box 1, Kensington, NSW 2033, Australia.

(C) Copyright Australian Mathematical Society 1988, Serial-fee code 0334-2700/88 
it still seems worthwhile to examine the modulation of short waves riding on a basic irrotational flow, if only to clarify and summarise this case before further extensions are contemplated. We shall show that there are two distinct cases to be considered, depending on whether the basic flow can be classified as a deepwater current, or a shallow-water current. This distinction, based on whether the vertical scale of the basic flow is comparable with the horizontal modulation scale or is much shorter, has not generally been recognised and results in some differences in detail between the two cases. Further, we shall show that some of the existing results in the literature can be generalised, and in some cases hold under wider hypotheses than the original derivations would have suggested.

In the remainder of this introductory section we shall present the basic equations of motion and the wave action conservation equation. Then in Section 2 we develop the theory for the case when the basic flow is a deep-water current and in Section 3 describe some applications. In Section 4 we describe the other case when the basic flow is a shallow-water current.

The equation of motion for an inviscid incompressible fluid of constant density, when the flow is irrotational, can be described in terms of a velocity potential $\phi(\mathbf{x}, z, t)$, where $\mathbf{x}$ is a horizontal co-ordinate, $z$ is the vertical co-ordinate and $t$ is the time. The fluid velocity is $(\mathbf{u}, w)$ where $\mathbf{u}$ is horizontal and $w$ is vertical.

$$
\mathbf{u}=\boldsymbol{\nabla} \phi, \quad w=\phi_{\boldsymbol{z}},
$$

and

$$
\nabla^{2} \phi+\phi_{z z}=0
$$

where $\boldsymbol{\nabla}$ denotes the horizontal gradient operator. The free surface is denoted by $z=\varsigma(\mathbf{x}, t)$, and the rigid bottom boundary by $z=-h(\mathbf{x})$. The boundary conditions are

$$
\begin{gathered}
\varsigma_{t}+\mathbf{u} \cdot \nabla \varsigma=w, \quad \text { on } z=\varsigma, \\
g \varsigma+\gamma \nabla \cdot \eta+\frac{1}{2}\left(|\mathbf{u}|^{2}+w^{2}\right)+\phi_{t}=-P_{0}, \quad \text { on } z=\varsigma,
\end{gathered}
$$

and

$$
w+\mathbf{u} \cdot \boldsymbol{\nabla} h=0, \quad \text { on } z=-h,
$$

where

$$
\eta=\left(-\nabla_{\zeta}, 1\right)\left(1+|\nabla \zeta|^{2}\right)^{-1 / 2} .
$$

Here $\eta$ is the unit normal to the free surface, while $g$ is the acceleration due to gravity, $\gamma$ is the surface tension coefficient and $P_{0}$ is an applied surface pressure.

To obtain the wave action conservation equation, we first suppose that $\phi(\mathbf{x}, z, t ; \theta)$ and $\varsigma(\mathbf{x}, t ; \theta)$ depend smoothly on a phase parameter $\theta$ and are periodic in $\theta$, with period $2 \pi$. We then define $<--->$ to be an average over one period in $\theta$. In practice the averaging operation can be identified as a local average over one wavelength, or over one period in time. For a general account of 
this procedure see Andrews and McIntyre $[1,2]$ or Grimshaw [7]. By multiplying (1.1b) by $\phi_{\theta}$, it follows that

$$
\nabla \cdot\left(\phi_{\theta} \nabla \phi\right)+\left(\phi_{\theta} \phi_{z}\right)-\left(\frac{1}{2}|\mathbf{u}|^{2}+\frac{1}{2} w^{2}\right)_{\theta}=0
$$

and

$$
\nabla \cdot\left\langle\phi_{\theta} \nabla \phi\right)+\left(\phi_{\theta} \phi_{z}\right)_{z}=0 .
$$

This last equation is the local form of wave action conservation. A more useful expression is the global form obtained by first integrating (1.3a) with respect to $z$, applying the boundary conditions $(1.2 \mathrm{a}, \mathrm{b}, \mathrm{c})$ and then averaging. The result is

$$
A_{t}+\nabla \cdot \mathrm{B}=0
$$

where

$$
A=\left\langle\varsigma_{\theta} \phi(\mathbf{x}, \varsigma, t)\right\rangle,
$$

and

$$
\mathbf{B}=\left\langle\int_{-h}^{\varsigma} \phi_{\theta} \nabla \phi d z\right\rangle-\gamma\left(\varsigma_{\theta} \eta_{H}\right\rangle
$$

Here $\eta_{H}$ is the horizontal component of $\eta(1.2 \mathrm{~d})$. These equations are formally exact, with no necessary restriction on either amplitude or length scale. In the absence of surface tension $(\gamma=0)$ these equations can be shown to be equivalent to those obtained by Whitham $[17,18]$ using an averaged variational principle, but the form given here appears not to be very widely known.

\section{Basic flow is a deep-water current: formulation}

Let $\varepsilon$ be a small parameter which measures the ratio of the short-wave wavelength to the length scale of the basic wave. Then, to describe the basic flow, we introduce the stretched space and time variables $\mathbf{X}$ and $\tau$ by

$$
\mathrm{X}=\varepsilon \mathbf{x}, \quad Z=\varepsilon z, \quad \tau=\varepsilon^{1 / 2} t .
$$

The basic flow is then described by a velocity field $\varepsilon^{-1 / 2} \mathbf{U}(\mathbf{X}, \mathbf{Z}, \tau)$, $\varepsilon^{-1 / 2} W(\mathbf{X}, Z, \tau)$, a velocity potential $\varepsilon^{-3 / 2} \Phi(\mathbf{X}, \mathbf{Z}, \tau)$, and a free surface displacement $\varepsilon^{-1} S(\mathbf{X}, \mathbf{Z}, \tau)$. On substituting these variables into $(1.1 \mathrm{a}, \mathrm{b})$ it follows that

$$
\mathbf{U}=\nabla_{\mathbf{X}} \Phi, \quad W=\Phi_{Z}
$$

and

$$
\nabla_{\mathbf{X}}^{2} \Phi+\Phi_{Z Z}=0
$$

while the boundary conditions $(1.2 \mathrm{a}, \mathrm{b}, \mathrm{c})$ become

$$
D S / D \tau=W, \quad \text { on } Z=S,
$$




$$
\begin{gathered}
g S+\varepsilon^{2} \gamma \nabla \mathbf{x} \cdot \mathbf{n}+\Phi_{\tau}+1 / 2\left(|\mathbf{U}|^{2}+W^{2}\right)=-P_{0}, \quad \text { on } Z=S, \\
W+\mathbf{U} \cdot \nabla_{\mathbf{X}} H=0, \quad \text { on } Z=-H,
\end{gathered}
$$

where

$$
D / D \tau=\partial / \partial \tau+\mathbf{U} \cdot \nabla_{\mathbf{X}}
$$

and

$$
\mathbf{n}=\cos \beta\left(1,-\nabla_{\mathbf{X}} S\right), \quad \cos \beta=\left(1+\left|\nabla_{\mathbf{X}} S\right|^{2}\right)^{-1 / 2} .
$$

Here the depth $h$ has been rescaled to $\varepsilon^{-1} H(X)$. Note that $\mathbf{n}$ is the unit normal to the free surface and $\beta$ is the angle between the normal to the free surface and the vertical. $P_{0}$ is an applied surface pressure. Equations $(2.2 \mathrm{a}, \mathrm{b})$ and $(2.3 \mathrm{a}, \mathrm{b}, \mathrm{c})$ describe a finite-amplitude deep-water wave or current. Relative to this basic flow we now introduce the perturbed velocity field $\mathbf{u}(\mathbf{x}, z, t)$, the perturbed velocity potential $\phi(\mathbf{x}, z, t)$ and the perturbed free surface displacement $\zeta(\mathbf{x}, t)$. Note that the total velocity field is $\varepsilon^{-1 / 2} U+u$, with similar expressions for the total velocity potential and the total free surface displacement, and that the variables $\mathbf{u}, \phi$ and $\varsigma$ now represent the perturbed fields, rather than the total fields as in Section 1. On substituting the total fields into $(1.1 \mathrm{a}, \mathrm{b})$ it follows that

$$
\mathbf{u}=\boldsymbol{\nabla} \phi, \quad w=\phi_{z},
$$

and

$$
\nabla^{2} \phi+\phi_{z z}=0 .
$$

The linearised free-surface boundary conditions $(1.2 \mathrm{a}, \mathrm{b})$ are

$$
\begin{aligned}
D_{\zeta} / D t+\mathbf{u} \cdot \nabla_{\mathbf{X}} S+\varepsilon^{1 / 2} \varsigma \nabla_{\mathbf{X}} \cdot \mathrm{U}_{0}=w, \quad \text { on } z & =\varepsilon^{-1} S, \\
g_{0} \varsigma-\gamma \nabla \cdot\left\{\cos \beta\left(\nabla \zeta-\left(\mathbf{n} \cdot \nabla_{\zeta}\right) \mathbf{n}\right)\right\}+(D / D t) \phi\left(\mathbf{x}, \varepsilon^{-1} S, t\right) & =0, \quad \text { on } z=\varepsilon^{-1} S
\end{aligned}
$$

where

$$
\begin{gathered}
\mathbf{U}_{0}=\mathbf{U}(\mathbf{X}, S, \tau), \\
g_{0}=g+\left(D^{2} S / D \tau^{2}\right),
\end{gathered}
$$

and

$$
D / D t=\partial / \partial t+\varepsilon^{-1 / 2} \mathrm{U}_{0} \cdot \nabla .
$$

Note that $\mathbf{U}_{0}$ is the basic horizontal current evaluated at the free surface, and $g_{0}$ is the effective vertical acceleration due to gravity experienced by the short waves. The bottom boundary condition $(1.2 \mathrm{c})$ becomes

$$
w+\mathbf{u} \cdot \nabla_{\mathbf{X}} H=0, \quad \text { on } z=-\varepsilon^{-1} H .
$$

As a preliminary to solving these equations, we introduce the new coordinate

$$
\eta=z-\varepsilon^{-1} S
$$


in place of $z$. We prefer the non-orthogonal curvilinear co-ordinate system $(\mathbf{x}, \eta)$ to the more obvious curvilinear co-ordinate system which has one co-ordinate normal to the free surface, as the latter would necessitate the introduction of intrinsic co-ordinates on the free surface. We believe it is more convenient to retain the conventional Cartesian co-ordinates to describe the free surface. In effect we are choosing to parameterise the free surface with the horizontal Cartesian co-ordinates $\mathrm{X}=(X, Y)$ rather than with a pair of co-ordinates intrinsic to the free surface, say $\sigma=\left(\sigma_{1}, \sigma_{2}\right)$. However the free surface can always be represented in the form $\mathbf{X}=\mathbf{X}(\sigma)$ and then a transformation of variables from $\mathrm{X} \leftrightarrow \sigma$ would enable all our results (e.g. (2.13), (2.22a) below) to be expressed in terms of $\sigma$. Proceeding, Laplace's equation becomes

$$
\nabla^{2} \phi+\sec ^{2} \beta \phi_{\eta \eta}-2 \nabla \mathbf{x} S . \nabla \phi_{\eta}-\varepsilon \nabla_{\mathbf{X}}^{2} S \phi_{\eta}=0
$$

while the free-surface boundary conditions $(2.5 \mathrm{a}, \mathrm{b})$ become

$$
\begin{array}{ll}
D_{\zeta} / D t+\nabla \phi \cdot \nabla_{\mathbf{X}} S+\varepsilon^{1 / 2} \zeta \nabla_{\mathbf{X}} \cdot \mathbf{U}_{0}=\sec ^{2} \beta \phi_{\eta}, & \text { on } \eta=0, \\
g_{0}-\gamma \nabla \cdot\left\{\cos \beta\left(\nabla_{\zeta}-\left(\mathbf{n} \cdot \nabla_{\zeta}\right) \mathbf{n}\right)\right\}+D \phi / D t=0, & \text { on } \eta=0 .
\end{array}
$$

The bottom boundary condition (2.6) becomes

$$
\left(1-\nabla_{\mathbf{x}} S \cdot \nabla_{\mathbf{x}} H\right) \phi_{\eta}+\nabla \phi \cdot \nabla_{\mathbf{x}} H=0, \quad \text { on } \eta=-\varepsilon^{-1}(H+S) .
$$

Next we seek solutions which describe modulated waves. Thus we put

$$
\begin{gathered}
\varsigma=a(\mathbf{x}, \tau) \exp (i \theta)+* \\
\phi=f(\mathbf{x}, \eta, \tau) \exp (i \theta)+*
\end{gathered}
$$

where

$$
\theta=(1 / \varepsilon) \Theta(\mathbf{X}, \tau)
$$

The local frequency $\omega$, and local wavenumber $\kappa$ are defined by

$$
\omega=-\varepsilon^{-1 / 2} \Theta_{\tau}, \quad \kappa=\nabla_{\mathbf{x}} \Theta,
$$

and satisfy the kinematic equation

$$
\kappa_{\tau}+\varepsilon^{1 / 2} \nabla_{\mathbf{x}} \omega=0,
$$

which describes conservation of wave crests. Before proceeding, we note that the ansatz $(2.11 \mathrm{a}, \mathrm{b}, \mathrm{c})$ excludes the possibility of an instability in the basic wave. It has been shown by Hasselmann [8] that high wavenumber instabilities of a periodic basic wave are due to an $N$ th order resonance, where $N$ is a large integer. In our terminology, $N$ scales with $\varepsilon^{-1 / 2}$ and the instabilities have a growth rate of $O\left(\alpha^{N}\right)$ on the $t$-time scale, and a wavenumber bandwidth of $O\left(\alpha^{N}\right)$, where $\alpha$ is a measure of the basic wave amplitude. In the asymptotic limit $\varepsilon \rightarrow 0$, these instabilities are insignificant and are ignored here. 
On substituting (2.11b) into Laplace's equation (2.8) we find that

$$
\sec ^{2} \beta f_{\eta \eta}-2 i \kappa . \nabla S f_{\eta}-\kappa^{2} f+O(\varepsilon)=0,
$$

where $\kappa=|\kappa|$, and here, and henceforth, we have omitted the subscript $\mathbf{X}$ from the horizontal operator $\nabla$ since, from this point on, no confusion should arise. The solution of (2.14) is

$$
f=b(\mathbf{X}, \tau) \exp (\lambda \eta)+O(\varepsilon)
$$

where

$$
\lambda=\left(i \kappa \cdot \nabla S+\kappa^{\prime}\right) \cos ^{2} \beta
$$

and

$$
\kappa^{\prime}=\left\{\kappa^{2} \sec ^{2} \beta-(\kappa \cdot \nabla S)^{2}\right\}^{1 / 2} .
$$

The boundary condition (2.10) is not satisfied by this solution, but the error involved is only $O\left(\exp \left\{-\lambda \varepsilon^{-1}[H+S]\right\}\right)$, and can be ignored since $\kappa^{\prime}$ is positive. In interpreting this solution we recall that $\mathbf{n}(2.3 \mathrm{e})$ is the unit normal to the interface, and put

$$
\nu=\{\boldsymbol{\kappa}-\mathbf{n}(\boldsymbol{\kappa} . \mathbf{n})\}\left(\kappa^{2}-(\boldsymbol{\kappa} . \mathbf{n})^{2}\right)^{-1 / 2},
$$

and

$$
\kappa_{S}=\kappa \cdot \nu=\kappa^{\prime} \cos \beta .
$$

Here $\boldsymbol{\nu}$ is the unit vector tangent to the interface in the place of $\mathbf{n}$ and $\boldsymbol{\kappa}$, and so $\kappa_{S}$ is the component of the wavenumber along the interface. Further, substituting (2.15a) into (2.11b), it follows that

$$
\phi=b(\mathbf{X}, \tau) \exp \left\{\left(i \Theta^{\prime} / \varepsilon\right)+\kappa^{\prime} \eta \cos ^{2} \beta\right\}+*+O(\varepsilon),
$$

where

$$
\Theta^{\prime}=\theta+\varepsilon \eta \kappa \cdot \nabla S \cos ^{2} \beta,
$$

is the total phase. It can now be shown that

$$
\left(\nabla \Theta^{\prime}, \Theta_{Z}^{\prime}\right)=\kappa_{S} \nu, \quad \text { on } \eta=0,
$$

and is oriented along the interface with magnitude $\kappa_{S}$ as expected. Also

$$
\partial / \partial n\left\{\kappa^{\prime} \eta \cos ^{2} \beta\right\}=\kappa_{S}, \quad \text { on } \eta=0,
$$

which confirms that the rate of decay normal to the interface is $\kappa_{S}$.

The expressions (2.11a) and (2.15a) are now substituted into the boundary conditions $(2.9 \mathrm{a}, \mathrm{b})$. It follows that

$$
\begin{gathered}
-i \sigma a=\kappa^{\prime} b+O\left(\varepsilon^{1 / 2}\right), \\
\left(g_{0}+\gamma \cos ^{3} \beta \kappa^{\prime 2}\right) a=i \sigma b+O\left(\varepsilon^{1 / 2}\right),
\end{gathered}
$$

where

$$
\sigma=\omega-\varepsilon^{-1 / 2} \kappa \cdot U_{0}
$$


Hence we obtain the local dispersion relation

$$
\sigma^{2}=g_{0} \kappa^{\prime}+\gamma \cos ^{3} \beta \kappa^{\prime 3},
$$

which, together with the kinematic equation (2.13) determines the variation of the phase $\theta$. Note that the leading order term in the frequency $\omega$ is the Dopplershift term which scales with $\varepsilon^{-1 / 2}$, rather than the intrinsic frequency $\sigma$, which is $O(1)$. These resulits agree with those of Phiilips [ib̂], who considered the case when the basic flow is steady and two-dimensional. Note that in this case $\kappa_{2}=\kappa^{\prime} \cos \beta=\left\{k^{2} \cos ^{2} \beta+l^{2}\right\}^{1 / 2}$ where $\kappa=(k, l)$ and the basic flow is in the $x$-direction. The local dispersion relation $(2.21)$ is clearly recognisable as the deep-water gravity wave dispersion relation, since $g_{0}(2.5 \mathrm{~d})$ is the effective acceleration due to gravity, and from (2.16) $\kappa^{\prime} \cos \beta$ is the effective wavenumber magnitude. In Section 3(a) we give more details of this special case.

The amplitude variation is now determined by considering the higher-order terms in $(2.14)$ and $(2.20 \mathrm{a}, \mathrm{b})$. The resulting analysis is quite lengthy but routine, and the details are omitted. The result is

$$
\begin{gathered}
D A / D \tau+A \nabla \cdot \mathrm{U}_{0}+\varepsilon^{1 / 2} \nabla \cdot(\mathrm{V} A)=O(\varepsilon) ; \\
D / D \tau(\arg a)+\varepsilon^{1 / 2} \mathrm{~V} \cdot \nabla(\arg a)=\varepsilon^{1 / 2}\left(\kappa^{\prime} / \sigma\right) M+O(\varepsilon),
\end{gathered}
$$

where

$$
A=\left(2 \sigma / \kappa^{\prime}\right)|a|^{2}
$$

and

$$
\mathbf{V}=\nabla_{\kappa} \sigma .
$$

Thus $\mathbf{V}$ is the intrinsic group velocity, and is given by

$$
\mathbf{V}=\left(2 \sigma \kappa^{\prime}\right)^{-1}\left(g_{0}+3 \gamma \kappa^{\prime 2} \cos ^{3} \beta\right)\left(\kappa \sec ^{2} \beta-(\kappa . \nabla S) \nabla S\right) .
$$

We note that $\mathbf{V}$ is horizontal and parallel to the horizontal component of $\nu$, but is not necessarily parallel to $\kappa$. Also the magnitude $|V|$, and its components $V . \nu$ and $V . n$ are given respectively by

$$
\begin{gathered}
|\mathbf{V}|=\left(2 \sigma \kappa^{\prime}\right)^{-1}\left(g_{0}+3 \gamma \kappa^{\prime 2} \cos ^{3} \beta\right)\left(\kappa^{\prime 2} \sec ^{2} \beta-(\kappa . \nabla S)^{2}\right)^{1 / 2} \\
\mathbf{V} . \nu=|\mathbf{V}|\left(1-(\kappa \cdot \nabla S)^{2} \cos ^{2} \beta\left(\kappa^{\prime}\right)^{-2}\right)^{1 / 2},
\end{gathered}
$$

and

$$
\mathbf{V} . \mathbf{n}=-|\mathbf{V}|(\boldsymbol{\kappa} . \nabla S) \cos \beta\left(\kappa^{\prime 2} \sec ^{2} \beta-(\boldsymbol{\kappa} \cdot \nabla S)^{2}\right)^{-1 / 2} .
$$

$A$ is the wave action density to leading order, and may be computed from (1.4b). Indeed the simplest derivation of $(2.22 \mathrm{a})$ is to use the wave action equation (1.4a), with $A$ and $B$ computed to leading order from $(1.4 b, c)$. However $(2.22 b)$, which gives phase information, cannot be computed from the wave action equation, and requires the more detailed analysis from $(2.14)$ and $(2.20 \mathrm{a}, \mathrm{b})$. The argument of 
$a$ can be regarded as an $O(\varepsilon)$ correction to the phase $\theta(2.11 \mathrm{c})$. The $O\left(\varepsilon^{1 / 2}\right)$ term in $(2.22 \mathrm{~b})$ is given by

$$
\begin{aligned}
M= & \left(2 \kappa^{\prime 2} \cos ^{4} \beta\right)^{-1}\left\{\kappa \cdot \nabla\left(\cos ^{2} \beta \kappa \cdot \nabla S\right)-\nabla S \cdot \nabla\left(\kappa^{2} \cos ^{2} \beta\right)\right\} \\
& +\left(\kappa^{\prime} / 4 \sigma^{2}\right)^{-1}\left\{\left(\nabla \cdot \mathrm{U}_{0}\right)^{2}-\left(\frac{\kappa^{\prime}}{\sigma} \frac{D}{D \tau}\left(\frac{\kappa^{\prime}}{\sigma}\right)\right)^{2}\right\} \\
& -\frac{1}{2} \frac{D}{D \tau}\left\{\frac{\kappa^{\prime}}{\sigma^{2}} \nabla \cdot \mathrm{U}_{0}+\frac{1}{\sigma} \frac{D}{D \tau}\left(\frac{\kappa^{\prime}}{\sigma}\right)\right\} .
\end{aligned}
$$

The wave action equation (2.22a) can be recast in terms of wave energy $E$, where

$$
E=\sigma A=2\left(g_{0}+\gamma \cos ^{3} \beta \kappa^{2}\right)|a|^{2} .
$$

Substituting (2.25) into (2.22a) we find that

$$
D E / D \tau+E \nabla \cdot \mathbf{U}_{0}+\varepsilon^{1 / 2} \nabla \cdot(\mathbf{V} E)+\frac{E}{\sigma}\left\{\boldsymbol{\kappa}\left(\mathbf{V} \cdot \nabla \mathbf{U}_{0}\right)-(D / D \tau)_{e} \sigma\right\}=O(\varepsilon) .
$$

Here $(D / D \tau)_{e}$ denotes the derivative of $\sigma(2.21)$ with both $\kappa$ and $\mathbf{U}_{0}$ held fixed, that is the derivative with respect to the dependence of $\sigma$ on $D^{2} S / D \tau^{2}$ and $\nabla S$. From the second to last term in (2.27) we can identify $E \kappa V \sigma^{-1}$ as a radiation stress tensor. The last term in (2.27) is atypical, in that it cannot generally be expressed in the form of a stress tensor acting on a velocity gradient. It vanishes in the limit when the basic flow has a small free surface slope $(\beta \rightarrow 0)$. In this same limit (2.27) agrees with the wave energy equations derived by LonguetHiggins and Stewart $[10,11]$. Further details of this limit will be described in the next section.

\section{Basic flow is a deep-water current: applications}

The equations governing the modulation of the short waves are the kinematic equation (2.13) for the conservation of wave crests, the Doppler-shift expression (2.20c), the local dispersion relation (2.21) and the wave action equation (2.22a). The scaling for the basic flow introduced at the beginning of Section 2 shows that the basic flow has an amplitude $\varepsilon^{-1}$ and a length scale $\varepsilon^{-1}$ so that $\beta$, the slope of the free surface, is generally finite and scales with 1 . The short waves have a characteristic amplitude $\alpha_{S}$ and a characteristic wavenumber $\kappa_{S}$, so that the slope of the short wave is $O\left(\alpha_{S} \kappa_{S}\right)$. Since the equations $(2.5 \mathrm{a}, \mathrm{b})$ for the short waves have been linearised, and the derivation of the modulation equations requires the retention of $O(\varepsilon)$ terms, the modulation equations are valid for $\alpha_{S} \kappa_{S} \ll \varepsilon$. The subsequent discussion will be divided into two cases, depending on whether $\beta$, the slope of the free surface for the basic flow, is finite or infinitesimal $\left(O\left(\varepsilon^{1 / 2}\right)\right)$. 
(a) $\beta$ finite: In this case the basic flow retains the scaling introduced in Section 2. The modulation equations become

$$
\begin{gathered}
\partial \kappa / \partial \tau+\nabla\left(\kappa . \mathbf{U}_{0}\right)=-\varepsilon^{1 / 2} \nabla \sigma \\
\partial A / \partial \tau+\nabla \cdot\left(\mathbf{U}_{0} A\right)=-\varepsilon^{1 / 2} \nabla \cdot(\mathbf{V} A) .
\end{gathered}
$$

Higher-order terms will not be displayed henceforth. If the $O\left(\varepsilon^{1 / 2}\right)$ terms on the right-hand side are omitted, these equations are particulariy simpie, as then the only feature of the basic flow that is needed is $U_{0}$, the horizontal current evaluated at the free surface. Equations $(3.1 \mathrm{a}, \mathrm{b})$ then determine $\kappa$ and $A$ respectively; with $\kappa$ known the local dispersion relation determines $\sigma$. These reduced equations are valid provided only that $\alpha_{S} \kappa_{S} \ll \varepsilon^{1 / 2}$. However, in general it is useful to retain the $O\left(\varepsilon^{1 / 2}\right)$ terms in $(3.1 \mathrm{a}, \mathrm{b})$ as these are the terms which contain $\mathbf{V}$, and hence the information on the intrinsic speed of propagation for the short-wave modulations.

Now let us consider the special case when the basic flow is a two-dimensional progressing wave of speed $c$. Thus $H$ is constant, and

$$
\mathbf{U}_{0}=U(X-c \tau) \mathbf{i}, \quad S=S(X-c \tau) \text { and } P_{0}=P_{0}(X-c \tau),
$$

where $\boldsymbol{I}$ is a unit vector in the $X$-direction. Subsequent simplifications in the basic flow equations allow us to simplify (2.21) to

$$
\sigma^{2}=\kappa_{S} \cos \beta\left(g+(U-c)^{2} S_{X X}-S_{X} P_{0 X}\right)+\kappa_{S}^{3},
$$

where we recall that $\kappa_{S}$ is the wavenumber component along the interface. If $\kappa=(k, l)$ then from $(2.15 \mathrm{c})$, and $(2.16)$ it follows that

$$
\kappa_{S}=\left\{k^{2} \cos ^{2} \beta+l^{2}\right\}^{1 / 2} .
$$

Also we note that $S_{X X} \cos ^{3} \beta$ is the curvature of the free surface of the basic flow. If we seek solutions of $(3.1 \mathrm{a}, \mathrm{b})$ which are functions only of $(X-c \tau)$ then it follows that $l$ is constant, and

$$
\begin{gathered}
k(U-c)+\varepsilon^{1 / 2} \sigma=\text { constant } \\
A\left\{(U-c)+\varepsilon^{1 / 2} V\right\}=\text { constant }
\end{gathered}
$$

where $V=V . i$ is the $x$-component of group velocity. For the case when the basic flow is a free wave $\left(P_{0}=0\right)$, these results agree with those of Phillips [16] who derived them using more heuristic and physical arguments. In making the comparison, note that the wavenumber component along the interface is $\kappa_{S}$, that in a frame of reference moving with the flow, $(U-c) \sec \beta$ is the basic current along the interface, and that here $|a|$ is the wave amplitude measured vertically, whereas in Phillips [16] the wave amplitude is measured normal to the interface. The present results thus extend those of Phillips [16] to include forced waves $\left(P_{0} \neq 0\right)$. Longuet-Higgins and Stewart [11] derived analogous equations to (3.3) 
and $(3.5 \mathrm{a}, \mathrm{b})$ for the case when the basic flow is a steady current $(c=0)$, with no applied forcing $\left(P_{0}=0\right)$, under the additional restriction that the free-surface slope is small $(\beta \rightarrow 0)$. A noteworthy special case of our results is when the basic flow is a steady forced current, with no surface displacement. Thus $c=0, S=0$, and from the basic flow equations $(2.3 \mathrm{a}-\mathrm{e}), W=0$ at $S=0$ and $P_{0}=-U^{2} / 2$. It follows that then $\beta=0$ and (3.3) is replaced by $\sigma^{2}=g \kappa S+\gamma \kappa_{S}^{3}$. Equations $(3.5 \mathrm{a}, \mathrm{b})$ then agree exactly with the results of Longuet-Higgins and Stewart [11] indicating that their results are also valid for this class of forced current. In general, however, equations $(3.5 \mathrm{a}, \mathrm{b})$ must be used with the full dispersion relation (3.3). Equation (3.5a) is an algebraic equation which determines $k$ (or $\kappa_{S}$ ), and then $A$ is found from (3.5b), with $|a|$ then given by (2.22c). Since Phillips [16] and more recently Longuet-Higgins [12,13], have given an extensive discussion of the solutions when $P_{0}=0$, we shall not discuss this case further.

Another special case of some interest occurs when the basic flow is a spatiallyvarying steady current so that $\mathbf{U}_{0}=\mathbf{U}_{0}(\mathbf{X})$. The solution of (3.1a) is then

$$
\mathbf{U}_{0} \cdot \nabla \Theta+\varepsilon^{1 / 2} \sigma=\omega_{0},
$$

where $\omega_{0}$ is a constant, and we recall from (2.12) that $\kappa=\nabla \theta$. Ignoring the $O\left(\varepsilon^{1 / 2}\right)$ term in (3.6), the solution of (3.6) is $\Theta=\omega_{0} s+\Theta_{0}(\psi)$ where $s$ is a timelike variable along the particle trajectories of the basic flow. These are described by $\mathbf{X}=\mathbf{X}(s, \psi)$, where $\psi$ is a co-ordinate which labels each trajectory. Also $\Theta_{0}(\psi)$ is the initial phase on each trajectory. Solving for $s$ and $\psi$ as functions of $\mathbf{X}$ then gives $\Theta=\Theta(\mathbf{X})$. Again ignoring the $O\left(\varepsilon^{1 / 2}\right)$ term the solution of (3.1b) is

$$
A J=\text { constant, }
$$

where $J$ is the Jacobian of the transformation from $(s, \psi)$ to $\mathbf{X}$. In the special case when $\mathrm{U}_{0}$ is non-divergent (i.e. $\boldsymbol{\nabla} \cdot \mathrm{U}_{0}=0$ ), we can identify $\psi$ as a stream function for $\mathbf{U}_{0}$, and then $J=1$; the wave action density $A$ is then constant along the streamlines of the basic flow.

(b) $\beta$ is $O\left(\varepsilon^{1 / 2}\right)$ : We shall now discuss the case when the basic flow has a small free-surface slope. This could be achieved simply by letting $\beta \rightarrow 0$ in the previous results. However, if $\beta \rightarrow 0$ while the current $U_{0}$ remains finite, then it is apparent from (2.3b) that the basic flow generally requires a pressure gradient to be maintained. In order to avoid this restrictive situation we shall adopt a rescaling of both $S$ and $\mathrm{U}_{0}$, thus we put

$$
S=\varepsilon^{1 / 2} \hat{S}, \quad \Phi=\varepsilon^{1 / 2} \hat{\Phi}
$$

so that

$$
\mathbf{U}=\varepsilon^{1 / 2} \hat{\mathbf{U}}=\varepsilon^{1 / 2} \nabla_{X} \hat{\Phi}, \quad W=\varepsilon^{1 / 2} \hat{W}=\varepsilon^{1 / 2} \Phi_{Z}
$$


$\hat{\Phi}(\mathbf{X}, Z)$ satisfies Laplace's equation (2.2b) and the boundary condition (2.3c), while the free-surface boundary conditions $(2.3 \mathrm{a}, \mathrm{b})$ become

$$
\begin{gathered}
\hat{S}_{\tau}=\hat{W}+O\left(\varepsilon^{1 / 2}\right), \quad \text { on } Z=0, \\
g \hat{S}+\hat{\Phi}_{\tau}=-\hat{P}_{0}+O\left(\varepsilon^{1 / 2}\right), \quad \text { on } Z=0,
\end{gathered}
$$

where $P_{0}=\varepsilon^{1 / 2} \hat{P}_{0}$. Thus, to $O\left(\varepsilon^{1 / 2}\right)$, the basic flow satisfies the linearised Equiationions for surface gravity waves. With this scaling $\beta$ is $O\left(\varepsilon^{1 / 2}\right)$, but it should be noted that the basic flow velocity field is finite, since we recall that the original velocity field was $\varepsilon^{-1 / 2} U$, and this is now $\hat{U}$. From $(2.15 \mathrm{c})$ we see that $\kappa^{\prime}=\kappa+O(\varepsilon)$ and the local dispersion relation (2.21) reduces to

$$
\sigma^{2}=g \kappa+\gamma \kappa^{3}+\varepsilon^{1 / 2} \kappa \hat{S}_{\tau \tau}+O(\varepsilon),
$$

while $(2.20 \mathrm{c})$ becomes,

$$
\sigma=\omega-\boldsymbol{\kappa} \cdot \hat{\mathbf{U}}_{0}+O\left(\varepsilon^{1 / 2}\right),
$$

where

$$
\hat{\mathbf{U}}_{0}=\hat{\mathbf{U}}(\mathbf{X}, Z=0, \tau) \text {. }
$$

Further, equations $(3.1 \mathrm{a}, \mathrm{b})$ become

$$
\begin{gathered}
\partial \kappa / \partial \tau+\varepsilon^{1 / 2} \nabla\left(\kappa . \hat{\mathbf{U}}_{0}+\sigma\right)=O(\varepsilon), \\
\partial A / \partial \tau+\varepsilon^{1 / 2} \nabla \cdot\left(\left(\hat{\mathbf{U}}_{0}+\mathrm{V}\right) A\right)=O(\varepsilon) .
\end{gathered}
$$

The modulation equations $(3.12 \mathrm{a}, \mathrm{b})$ can now be solved by expanding with respect to $\varepsilon^{1 / 2}$. Thus we put

$$
\begin{aligned}
& \kappa=\kappa_{0}(\mathbf{X})+\varepsilon^{1 / 2} \kappa_{1}+O(\varepsilon), \\
& A=A_{0}(\mathbf{X})+\varepsilon^{1 / 2} A_{1}+O(\varepsilon) .
\end{aligned}
$$

Here $\kappa_{0}(X)$ and $A_{0}(\mathbf{X})$ are determined from initial conditions. It follows that

$$
\begin{gathered}
\partial \kappa_{1} / \partial \tau+\nabla\left(\kappa_{0} . \hat{U}_{0}+\sigma_{0}\right)=0 \\
\partial A_{1} / \partial \tau+\nabla \cdot\left(\left(\hat{U}_{0}+V_{0}\right) A_{0}\right)=0
\end{gathered}
$$

where

$$
\sigma_{0}^{2}=g \kappa_{0}+\gamma \kappa_{0}^{3}
$$

and

$$
\mathrm{V}_{0}=\nabla_{\kappa_{0}} \sigma_{0}
$$

Equations $(3.14 \mathrm{a}, \mathrm{b})$ determine $\kappa_{1}$ and $A_{1}$ respectively. The wave amplitude $a$ is found from $(2.22 b, c)$. We put

$$
\begin{gathered}
|a|=b_{0}(\mathbf{X})+\varepsilon^{1 / 2} b_{1}+O(\varepsilon), \\
\arg a=\delta_{0}(\mathbf{X})+\varepsilon^{1 / 2} \delta_{1}+O(\varepsilon)
\end{gathered}
$$


where

$$
A_{0}=2 \sigma_{0} b_{0}^{2} / \kappa_{0}
$$

while $b_{0}(X)$ is determined from the initial conditions. It follows that

$$
2 b_{1} / b_{0}=\left(A_{1} / A_{0}\right)-\left(\sigma_{1} / \sigma_{0}\right)+\kappa_{1} \cdot \kappa_{0} / \kappa_{0}^{2}
$$

where

$$
\sigma_{1}=\mathrm{V}_{0} \cdot \kappa_{1}+\left(\kappa_{0} / 2 \sigma_{0}\right) \hat{S}_{\tau \tau}
$$

and

$$
\partial \delta_{1} / \partial \tau+\left(\hat{\mathbf{U}}_{0}+\mathbf{V}_{0}\right) \cdot \nabla \delta_{0}=0 .
$$

As an illustration of the solution procedure, let us again suppose that the basic flow is a two-dimensional progressing wave of speed $c$. Thus we put

$$
\hat{\mathbf{U}}_{0}=\hat{U}(X-c \tau) \mathbf{i}, \quad \hat{S}=\hat{S}(X-c \tau),
$$

and

$$
\hat{P}_{0}=\hat{P}_{0}(X-c \tau)
$$

If we assume that $\kappa_{0}=\left(k_{0}, l_{0}\right)$ and $A_{0}$ are constant, and seek solutions of (3.14a, b) which are functions of $(X-c \tau)$ above, it then follows that $l_{1}=0$, and

$$
k_{1} / k_{0}=\hat{U} / c \text {, and } A_{1} / A_{0}=\hat{U} / c \text {. }
$$

Further, $b_{1}$, and $\sigma_{1}$ are then given by $(3.16 \mathrm{a}, \mathrm{b})$ respectively, and $\delta_{1}=0$. If it is further assumed that the basic flow is a free wave (that is, $\hat{P}_{0}=0$ ) with wavenumber $\varepsilon L$, then

$$
\hat{U} / c=(\operatorname{coth} L H) L \hat{S},
$$

where we recall that $\varepsilon^{-1} H$ is the fluid depth, here assumed to be constant. It follows that

$$
2 \frac{b_{1}}{b_{0}}=\frac{U}{c}\left\{1+\frac{k_{0}^{2}}{2 \kappa_{0}^{2}} \frac{\left(g-\gamma \kappa_{0}^{2}\right)}{\left(g+\gamma \kappa_{0}^{2}\right)}+\frac{g \tanh ^{2} L H}{\left(g+\gamma \kappa_{0}^{2}\right)}\right\}
$$

and

$$
\frac{2 \sigma_{1}}{\sigma_{0}}=\frac{\hat{U}}{c}\left\{\frac{\left(g+3 \gamma \kappa_{0}^{2}\right)}{\left(g+\gamma \kappa_{0}^{2}\right)} \frac{k_{0}^{2}}{\kappa_{0}^{2}}-\frac{g \tanh ^{2} L H}{\left(g+\gamma \kappa_{0}^{2}\right)}\right\} .
$$

For the case when the modulation is also in the $X$-direction $\left(l_{0}=0\right)$, there is no forcing $\left(\hat{P}_{0}=0\right)$ and in the absence of surface tension $(\gamma=0)$, these results for $k_{1}$ and $b_{1}$ agree with those of Longuet-Higgins and Stewart [10]. However, it is worthwhile noting that the present results are valid when $\alpha_{S} \kappa_{S} \ll \varepsilon$ where we recall that $\alpha_{S} \kappa_{S}$ is a measure of the slope of the short waves and $\varepsilon$ is the ratio of the short-wave wavelength to the long-wave wavelength, whereas the derivation by Longuet-Higgins and Stewart [10] requires both $\alpha_{S} \kappa_{S} \ll \varepsilon$ and $\alpha_{L} \kappa_{S} \ll \varepsilon$, where $\alpha_{L}$ is the amplitude of the long wave. Here, of course, $\alpha_{L}$ scales with $\varepsilon^{-1 / 2}$, although the long-wave slope (that is $\beta$ ) is $O\left(\varepsilon^{1 / 2}\right)$. 
A simple extension of the above results is for the case when the short waves are described by a continuous narrow-banded spectrum at leading order, instead of being described by a discrete mode. In physical space we model this situation by again supposing that initially $\kappa_{0}$ is a constant, but that $b_{0}$ and hence $A_{0}$ are functions of $X$. Then $\boldsymbol{\kappa}$ is again given by (3.13a) with $\kappa_{1}=\left(k_{1}, 0\right)$ and $k_{1}$ given by $(3.18)$. However, $(3.13 \mathrm{~b})$ is now replaced by

$$
A=A_{0}\left(X-\varepsilon^{1 / 2} V_{0} \tau+\varepsilon^{1 / 2} \xi\right)+\varepsilon^{1 / 2} A_{1}+O(\varepsilon),
$$

where

$$
V_{0}=\mathbf{V}_{0} . \mathbf{i}=\left(g+3 \gamma \kappa_{0}^{2}\right) k_{0} / 2 \sigma_{0} \kappa_{0} .
$$

A simple calculation now shows that, to leading order, $A_{1}$ is again given by (3.18), $b$ is found from (3.16a), and $\xi$, given by

$$
\xi_{\tau}+\hat{U}(X-c \tau)=0,
$$

measures a displacement of the centre of the wave packet. For the case when the basic wave is a free wave (that is $\hat{P}_{0}=0$ ) with wavenumber $\varepsilon L$, the solution of $(3.22)$ is

$$
\xi=-\hat{S}_{X}(L \tanh L H)^{-1} .
$$

This last result agrees with that recently obtained by Craik [4] who used a completely different derivation, based on long-short wave interactions described in spectral space. While this latter approach can also, in principle, obtain results for wide-band spectrums as well, it appears to be immensely more complicated algebraically than the present theory.

Finally we note that the results (3.18) or $(3.21 \mathrm{a}, \mathrm{b})$ can be expressed in a slightly more general form directly from $(3.12 a, b)$. With the basic flow given by (3.17), and $\mathbf{k}=(k, l)$, it follows from (3.12a) that $l$ is constant, and $k$ is given by

$$
-k c+\varepsilon^{1 / 2}(k U+\sigma)=\text { constant } .
$$

The wave action density is then found from (3.12b), whose solution is

$$
A\left\{-c+\varepsilon^{1 / 2}(U+V)\right\}=\text { constant on the rays, } d x / d \tau=\varepsilon^{1 / 2}(U+V),
$$

where $V=\mathbf{V} . \mathbf{i}$, is the $X$-component of the group velocity. Expanding with respect to $\varepsilon^{1 / 2}$ then recovers the previous results.

\section{Basic flow is a shallow-water current}

The essential difference between this and the previous deep-water case is that the vertical scale of the basic flow is $O(1)$ instead of scaling with $\varepsilon^{-1 / 2}$. Hence we put

$$
T=\varepsilon t
$$


and let the basic flow be described by the velocity potential $\varepsilon^{-1} \Phi(\mathrm{X}, z, T)$, and a free surface displacement $S(\mathrm{X}, T)$. Note that, although we are using the same notation, $\Phi$ and $S$ differ in scaling from the corresponding quantities introduced at the beginning of Section 2 . On substituting the velocity potential into (1.1b), it follows that

$$
\varepsilon^{2} \nabla_{\mathbf{X}}^{2} \Phi+\Phi_{z z}=0
$$

and so

$$
\Phi(\mathbf{X}, z, T)=\Phi_{0}(\mathbf{X}, T)+O\left(\varepsilon^{2}\right),
$$

and the horizontal velocity $\mathbf{U}(\mathbf{X}, z, T)$ is given by

$$
\mathbf{U}(\mathbf{X}, z, T)=\mathbf{U}_{0}(\mathbf{X}, T)+O\left(\varepsilon^{2}\right),
$$

where

$$
\mathrm{U}_{0}=\nabla_{\mathbf{X}_{0}} \Phi_{0} .
$$

To leading order the horizontal velocity is independent of depth. Although $\mathbf{U}_{0}$ has been obtained by a different process here, it has essentially the same meaning as the $U_{0}$ defined in Section 2, that is it describes the surface current. On using the bottom boundary condition, the vertical velocity $W(\mathbf{X}, z, T)$ is given by

$$
W=-\varepsilon \nabla \mathbf{X} \cdot\left\{(z+h) \mathbf{U}_{0}(\mathbf{X}, T)\right\}+O\left(\varepsilon^{3}\right) .
$$

The free-surface boundary conditions $(1.2 \mathrm{a}, \mathrm{b})$ now give

$$
\begin{gathered}
S_{T}+\nabla_{\mathbf{X}} \cdot\left(D \mathbf{U}_{0}\right)=0 \\
g S+\Phi_{0 T}+\frac{1}{2}\left|\mathbf{U}_{0}\right|^{2}=-P_{0}+O\left(\varepsilon^{2}\right)
\end{gathered}
$$

where

$$
D=h+S \text {. }
$$

Together with (4.4b) these equations are, to leading order, the nonlinear shallowwater equations. Relative to this basic flow we introduce the perturbed velocity field $\mathbf{u}$, the perturbed velocity potential $\phi$, and the perturbed free-surface displacement $\varsigma$. Equations (2.4a, b) again hold, but the linearised free-surface boundary conditions $(1.2 \mathrm{a}, \mathrm{b})$ are now

$$
\begin{gathered}
D_{\zeta} / D t+\varepsilon \mathbf{u} \cdot \nabla_{\mathbf{X}} S+\varepsilon \zeta \nabla \cdot \mathrm{U}_{0}=w, \quad \text { on } z=S, \\
g \varsigma-\gamma \nabla^{2} \varsigma+(D / D t) \phi(\mathbf{x}, S, t)=O\left(\varepsilon^{2}\right), \quad \text { on } z=S .
\end{gathered}
$$

Of course these equations are just (2.5a-e) but with a different scaling for the basic flow. The bottom boundary condition (1.2c) becomes

$$
w+\varepsilon \mathbf{u} \cdot \nabla_{\mathbf{X}} h=0, \quad \text { on } z=-h(\mathbf{X}) .
$$

Next we seek solutions which describe modulated waves. Similarly to (2.11ac) we put

$$
\varsigma=a(\mathbf{X}, T) \exp (i \theta)+*
$$




$$
\phi=f(\mathbf{X}, z, T) \exp (i \theta)+*
$$

where

$$
\theta=(1 / \varepsilon) \Theta(\mathbf{X}, T)
$$

Note again that although we are using the same notation these expressions differ in their scaling from the corresponding expressions used in Section 2. The local frequency and wavenumber are defined by

$$
\omega=-\theta_{T}, \quad \kappa=\nabla_{\mathbf{X}} \boldsymbol{\theta},
$$

and satisfy the kinematic equation

$$
\kappa_{T}+\nabla_{\mathbf{X}} \omega=0 .
$$

On substituting (4.9b) into Laplace's equation (2.4b), and using the bottom boundary condition (4.8), we find that

$$
f=b(\mathrm{X}, T) \frac{\cosh \kappa(z+h)}{\cosh \kappa D}+O(\varepsilon) .
$$

The free-surface boundary conditions $(4.7 \mathrm{a}, \mathrm{b})$ then give

$$
\begin{gathered}
-i \sigma a=\kappa \tanh \kappa D b+O(\varepsilon), \\
\left(g+\gamma \kappa^{2}\right) a=i \sigma b+O(\varepsilon),
\end{gathered}
$$

where

$$
\sigma=\omega-\kappa \cdot \mathrm{U}_{0} .
$$

Hence we obtain the local dispersion relation

$$
\sigma^{2}=\left(g+\gamma \kappa^{2}\right) \kappa \tanh \kappa D,
$$

which, together with the kinematic equation (4.11) determines the variation of the phase $\theta$. The amplitude variation is now determined by considering the higher-order terms in (4.12) and (4.13a, b). Since this analysis is quite lengthy, but routine, the details are omitted. The result is

$$
\begin{gathered}
\partial A / \partial T+\nabla_{\mathbf{X}} \cdot\left(\left(\mathbf{U}_{0}+\mathbf{V}\right) A\right)=O(\varepsilon), \\
\partial / \partial T(\arg a)+\left(\mathbf{U}_{0}+\mathbf{V}\right) \cdot \nabla_{\mathbf{X}}(\arg a)=O(\varepsilon),
\end{gathered}
$$

where

$$
A=2 \sigma|a|^{2} /(\kappa \tanh \kappa D),
$$

and

$$
\mathbf{V}=\nabla_{\kappa} \sigma .
$$

Here $A$ is the wave action density to leading order, and may be computed from (1.4b). Indeed (4.15a) is most easily derived directly from (1.4a). However, $(4.15 \mathrm{~b})$, which gives phase information, cannot be computed directly from the wave-action equation. These results (that is (4.11), (4.14), (4.15a-d)) are, of 
course, well known and can be obtained as a special case of internal or surface waves propagating on a horizontal current (see, for instance Grimshaw, [6]). The wave-action equation (4.15a) can be recast in terms of wave energy $E$, where

$$
E=\sigma A=2\left(g+\gamma \kappa^{2}\right)|a|^{2} .
$$

Substituting (4.16) into (4.15a), we find that

$$
\partial E / \partial T+\nabla_{X} \cdot\left(\left(\mathbf{U}_{0}+\mathbf{V}\right) E\right)+(E / \sigma)\left\{\boldsymbol{\kappa} \cdot\left(\mathbf{V} \cdot \nabla \mathbf{U}_{0}\right)+D \partial \sigma / \partial D \nabla \cdot \mathbf{U}_{0}\right\}=O(\varepsilon)
$$

where

$$
2 \sigma \partial \sigma / \partial D=\left(g+\gamma \kappa^{2}\right) \kappa^{2} \operatorname{sech}^{2} \kappa D .
$$

In the absence of surface tension $(\gamma=0)$, equation (4.17a) is equivalent to that proposed by Longuet-Higgins and Stewart [11]. The equivalence between (4.15a) and (4.17a) was noted in the work of Whitham [17], Garrett [5] and in Bretherton and Garrett [3]. From the last term in (4.17a) we can identify $E(\kappa \mathrm{V}+D \partial \sigma / \partial D \mathrm{I}) \sigma^{-1}$ as a radiation stress tensor.

The equations governing the modulation of the short waves are the kinematic equation (4.11a), the Doppler-shift expression (4.13c), the local dispersion relation (4.14) and the wave-action equation (4.15a). The basic flow has a freesurface slope of $O\left(\alpha_{S} \kappa_{S}\right)$. Because of the neglect of the nonlinear terms in the short-wave equations, the modulation equations are valid for $\alpha_{S} \kappa_{S} \ll \varepsilon$. Note, however, that the basic flow has a finite current $U_{0}$ and a finite free-surface displacement $S$. Longuet-Higgins and Stewart $[10,11]$ have given an extensive discussion of the modulation of short waves by long waves, including the parameter regime we are now discussing. Hence we shall not give a detailed account of the various applications of the modulation equations, but will confine our account to the same special case discussed in Section 3 when the basic flow is a two-dimensional progressing wave of speed $c$. Thus $h$ is a constant, and we put (cf. (3.2))

$$
\mathbf{U}_{0}=U(X-c T) \mathbf{i}, \quad S=S(X-c T),
$$

and

$$
P_{0}=P_{0}(X-c T) .
$$

If we now seek solutions of the modulation equations which are functions only of $X-c T$, it follows that $l$ is constant, and

$$
k(U-c)+\sigma=\text { constant }
$$

where $\kappa=(k, l)$. Here $c$ is given by (4.14) and depends, inter alia, on $S$ (see (4.6c)). The wave action density is given by

$$
A\{(U-c)+V\}=\text { constant }
$$

where $V=V . i$ is the $X$-component of group velocity. The wave amplitude is obtained from $(4.15 \mathrm{c})$. 
Although the expressions (4.19) and (4.20) are appealingly simple, they are algebraically complex. In order to obtain explicit expressions for $k$ and $|a|$, we now make the further hypothesis that $|S| \ll h$ (or $|U| \ll c$ ). It follows that we may put $k=k_{0}+k_{1}$, where $k_{0}$ is a constant, and $\left|k_{1}\right| \ll\left|k_{0}\right|$. From (4.19) we can show that

$$
\left(c-V_{0}\right)\left(k_{1} / k_{0}\right)=\left(1+\left(h / k_{0} c\right)\left(\partial \sigma_{0} / \partial h\right) U\right)+\cdots,
$$

where $\sigma_{0}$ and $V_{0}$ are $\sigma$ and $V$ evaluated at $k_{0}$, and $\partial \sigma / \partial h$ is given by $(4.17 \mathrm{~b})$. For the wave amplitude we put $|a|=b_{0}+b_{1}$ where $b_{0}$ is a constant, and $\left|b_{1}\right| \ll b_{0}$. From (4.15c) it follows that

$$
2 b_{1} / b_{0}=\left(A_{1} / A_{0}\right)+\left(\sigma_{1} / \sigma_{0}\right)-2 \gamma k_{0} k_{1} /\left(g+\gamma k_{0}^{2}\right)+\cdots,
$$

where

$$
\sigma_{1}=V_{0} k_{1}+h\left(\partial \sigma_{0} / \partial h\right)(U / c)+\cdots,
$$

Next from (4.20) we can show that

$$
\left(c-V_{0}\right) A_{1} / A_{0}=U+V_{1}+\cdots,
$$

and

$$
V_{1}=\left(\partial^{2} \sigma_{0} / \partial k_{0}^{2}\right) k_{1}+h\left(\partial^{2} \sigma_{0} / \partial k_{0} \partial h\right)(U / c)+\cdots
$$

For the case when the modulation is also in the $X$-direction $\left(l_{0}=0\right)$, there is no forcing $\left(\hat{P}_{0}=0\right)$ and in the absence of surface tension $(\gamma=0)$ these results for $k_{1}$ and $b_{1}$ agree with those of Longuet-Higgins and Stewart [10].

A simple extension of the above results is for the case when the short waves are described by a continuous narrow-banded spectrum at leading order, instead of being described by a discrete mode. As in Section 3, we model this situation by supposing that $k$ is again given by (4.19), but that $A$ is initially given by $A_{0}(X)$; the initial value for $|a|$ then follows from (4.15c). The expression (4.21) for the wave-action density is now replaced by

$$
A\{(U-c)+V\}=\text { constant on the rays, } \quad d X / d T=U+V .
$$

For the case when $|S| \ll h$, or $|\mathrm{U}| \ll c$, it follows as before that $k_{0}$ is a constant and $k_{1}$ is given by (4.21). But $A_{0}$ (and hence $b_{0}$ ) is a function of $X$ and (4.24) reduces to

$$
A=A_{0}\left(X-V_{0} T+\xi\right)+A_{1}+\cdots,
$$

where

$$
\xi_{\tau}+V_{0} \xi_{X}+U+V_{1}+\cdots=0
$$

while $A_{1}$ is again given by (4.23a), and $b_{1}$ is given by (4.22a). As in Section 3, $\xi$ measures a displacement of the centre of the wave packet. 


\section{Summary and discussion}

In this account of the modulation of short waves by long waves or currents, two cases have been identified, depending on whether the basic flow can be classified as a deep-water current, or a shallow-water current. In both cases, the modulation equations consist of a local dispersion relation for the short waves, the kinematic equation for the conservation of wave crests and the wave action equation. The first two equations together determine the short-wave wavenumber, and then the wave-action equation determines the wave amplitude. One of the main differences between the two cases is that when the basic flow is a deep-water current, the short waves are effectively in deep water (that is do not feel the bottom) and their local dispersion relation (2.21) is strongly influenced by the slope of the free surface of the basic flow, which is generally finite whereas when the basic flow is a shallow-water current the short waves feel the bottom, but the local dispersion relation (4.14) is not directly affected by the slope of the free surface which is now small. Another main difference is that when the basic flow is a deep-water current, this current is generally much greater than the group velocity of the short waves, with the consequence that the kinematic equation (3.1a) is dominated by the Doppler-shift term in the frequency, while in the wave-action equation (3.1b), transport by the basic current dominates. In contrast, when the basic flow is a shallow-water current, the kinematic equation (4.11) and the wave-action equation (4.15a) adopt more conventional forms.

The applications described here can be regarded as extending the early work of Longuet-Higgins and Stewart $[10,11]$ on the modulation of short waves by a steadily progressing long wave, or by a steady current, both by clarifying the range of validity of their results and by including the effects of surface tension, and allowing for the basic flow to be forced by a surface pressure. In particular, the results obtained here show that it is not necessary to assume that the long wave has small amplitude, or that the basic current has small free-surface slope. These restrictions were removed by Phillips [16], and more recently by LonguetHiggins [12,13], and the present results agree with these authors, and indicate several possible extensions. One of these is to the case when the short waves are described by a narrow-banded spectrum, instead of a discrete mode (see (3.21a, b) and (3.22)). Another is to the case when the short waves are modulated by a spatially varying steady current (see (3.6) and (3.7)).

There are a number of limitations of the present work. One of these is the restriction that the short waves be described by linearised equations. However, as the work of Whitham $[17,18]$ indicates, a theory can be developed for finiteamplitude short waves for the case when the basic flow is a deep-water current, in which the modulation equations would be the local dispersion relation, the wave 
kinematic equation and the wave-action equation (Peregrine and Thomas, [15]). For the case when the basic wave is a shallow-water current, these equations would have to be supplemented by equations describing the wave-induced mean flow (see, for instance, Andrews and McIntyre [1, 2] and Grimshaw [7]). A more severe restriction is that to irrotational flow, as the effect of shear in the surface layer of the ocean is likely to be very significant for the modulation of short waves. For the case when the underlying shear flow can he characterised as a shallow-water current, this case has been discussed by Grimshaw [6] in a more general context. However, the case when the underlying shear flow can be characterised as a deep-water current has apparently yet to be considered. Nevertheless, in spite of these limitations, it is to be hoped that this account will be found useful in clarifying and summarising our existing understanding of the modulation of short waves by long waves or currents.

\section{Acknowledgement}

It is a pleasure to record the many useful discussions with Dr. A. D. D. Clark, whose visit to U.N.S.W. revived my interest in this topic, and whose comments on previous drafts of this manuscript were very valuable.

\section{References}

[1] D. G. Andrews and M. E. McIntyre, "An exact theory of nonlinear waves on a Lagrangian mean-flow", J. Fluid Mech. 89 (1978), 609-646.

[2] D. G. Andrews and M. E. McIntyre, "On wave-action and its relatives", J. Fluid Mech. 89 (1978), 647-664.

[3] F. P. Bretherton and C. J. R. Garrett, "Wavetrains in inhomogeneous moving media", Proc. Roy. Soc. London Ser A 302 (1969), 539-554.

[4] A. D. D. Craik, "Interaction of a short-wave field with a dominant long wave in deep water: derivation from Zakharov's spectral formulation", J. Austral. Math. Soc. Ser. B 29 (1988), 430-439.

[5] C. J. R. Garrett, "On the interaction between internal gravity waves and a shear flow", J. Fluid Mech. 34 (1968), 711-720.

[6] R. Grimshaw, "Mean flows induced by internal gravity wave packets propagating in a shear flow", Philos. Trans. Roy. Soc. London Ser A 292 (1979), 391-417.

[7] R. Grimshaw, "Wave action and wave-mean flow interaction, with application to stratified shear flows", Ann. Rev. Fluid Mech. 16 (1984), 11-44.

[8] D. E. Hasselman, "The high wavenumber instabilities of a Stokes wave", J. Fluid Mech. 83 (1979), 491-499.

[9] P. H. Le Blond and L. A. Mysak, Waves in the ocean, (Elsevier, Amsterdam, 1978).

[10] M. S. Longuet-Higgins and R. W. Stewart, "Changes in the form of short gravity waves on long waves and tidal currents", J. Fluid Mech. 8 (1960), 565-583. 
[11] M. S. Longuet-Higgins and R. W. Stewart, "The changes in amplitude of short gravity waves on steady non-uniform currents", J. Fluid Mech. 10 (1961), 529-549.

[12] M. S. Longuet-Higgins, "Surface wave interactions", 9th. Australasian Fluid Mechanics Conference, (1986) 29-34.

[13] M. S. Longuet-Higgins, "The propagation of short surface waves on longer gravity waves", J. Fluid Mech. 177 (1987), 293-306.

[14] C. C. Mei, The applied dynamics of ocean surface waves, (Wiley, New York, 1983).

[15] D. H. Peregrine and G. P. Thomas, "Finite-amplitude deep-water waves on currents", Philos. Trans. Roy. Soc. Ser. A 292 (1979), 371-390.

[16] O. M. Phillips, "The dispersion of short wavelets in the presence of a dominant long wave", J. Fluid Mech. 107 (1981), 465-485.

[17] G. B. Whitham, "Non-linear dispersion of water waves", J. Fluid Mech. 27 (1967), 309 412.

[18] G. B. Whitham, Linear and nonlinear waves, (Wiley, New York, 1974). 